\title{
Experiencia docente a través de la interacción con administraciones locales
}

\author{
José Sergio Palencia Jiménez ${ }^{a}$, Eric Gielen ${ }^{b}$, Asenet Sosa Espinosa ${ }^{c}$, Vicent Altur \\ Grau $^{d}$ \\ ${ }^{a}$ Profesor Asociado. Departamento de Urbanismo. Universitat Politècnica de València. \\ jpalenci@urb.upv.es; ${ }^{b}$ Profesor Contratado Doctor. Departamento de Urbanismo. Universitat \\ Politècnica de València. egielen@urb.upv.es, ${ }^{c}$ Profesora Titular de Escuela Universitaria. \\ Departamento de Urbanismo. Universitat Politècnica de València. assoes@urb.upv.es, ${ }^{\mathrm{d}}$ Profesor \\ Titular de Escuela Universitaria. Departamento de Urbanismo. Universitat Politècnica de València. \\ vialgr@urb.upv.es.
}

\section{Resumen}

La incorporación de la dimensión urbana en la política de la UE, a través de la Agenda Urbana, poniendo el foco sobre la ciudad en materia de sostenibilidad, reducción de emisiones de $\mathrm{CO}_{2}$, mejora de las condiciones mediambientales y la cohesión social, fundamenta la aparición, el contenido y desarrollo de la asignatura denominada Medio Ambiente y Desarrollo Sostenible, cursada en $4^{\circ}$ del Grado de Ingeniería de Obras Públicas, y que viene desarrollando, desde hace cinco años, trabajos aplicados sobre distintas ciudades de la Comunitat Valenciana, denominados Auditoría Ambiental.

Para ello se han utilizado metododologías activas de enseñanza aprendizaje que restan protagonismo al rol docente para trasladarlo al alumnado, que debe aplicar sus conocimientos previos en la elaboración de la auditoría, en conexión organizativa con el profesorado como tutor. Este ejercicio práctico ha sido posible realizarlo con la colaboración e implicación directa de diversas administraciones locales que han contribuido facilitando información y han estado dispuestas a recibir los resultados obtenidos por el alumnado durante una exposición pública, seguida de un debate entre técnicos y políticos de dicha administración.

Con esta experiencia, se pretende que el alumnado adquiera competencias relacionadas con la comprensión y uso del lenguaje propio de la ingeniería, con el aprendizaje autónomo de conocimientos y técnicas para la ingeniería civil, así como, desarrollar el pensamiento y análisis crítico de los procesos propios de la ingeniería, a la vez que desarrollan un ejercicio académico lo más próximo al futuro ejercicio profesional. 
Los procesos llevados a cabo y los resultados obtenidos a través de esta experiencia docente son presentados en esta comunicación.

Palabras clave: Aprendizaje activo, ingeniería civil, sostenibilidad, análisis crítico, auditoría ambiental.

\section{Introducción}

El estudiante de cuarto curso de grado es un estudiante maduro en cuanto a la adquisición de conocimientos, sin embargo en el desarrollo de trabajos docentes, próximos a la profesión, presenta carencias significativas en cuanto a la interpretación de resultados, uso del lenguaje y, particularmente,en su comunicación y transmisión compresiva a la sociedad civil.

En aras de potenciar las cuestiones planteadas en el programa formativo, planteamos en el Plan de Estudios de Ingeniería de Obras Públicas, implantado hace cinco años, una asignatura optativa que tiene por objeto introducir al estudiante en materias relacionadas con el desarrollo sostenible, a diferentes escalas territoriales, a fin de que pudieran desarrollar un trabajo denominado "Auditoría Ambiental" aplicado a una ciudad. Este trabajo debe entenderse como una práctica académica que trata de acercar al alumnado los a la práctica profesional, tal y como se ha señalado anteriormente, siendo el municipio objeto de la auditoría y laboratorio territorial que contribuirá a alcanzar los objetivos docentes fijados en la asignatura de Medio Ambiente y Desarrollo Sostenible, cursada en $4^{\circ}$ grado de OOPP.

El desarrollo de este trabajo académico ha permitido interactuar con diferentes municipios de la Comunitat Valenciana, a través de la exposición pública, por parte del alumnado participante, de cuatro de dichas auditorías, teniendo como interlocutores a las autoridades locales y técnicos municipales, cerrando de este modo el proceso formativo propuesto.

\section{Metodología}

La metodología de enseñanza-aprendizaje que se ha aplicado en la materia descrita, supone la asunción por parte del alumnado de un rol activo y de autoaprendizaje en todo el proceso, puesto que el nuevo ingeniero civil no debe ser un profesional anclado en la elaboración y cálculo de fórmulas e indicadores, sino que ese proceso forme parte de su formación en análisis integral, autoresponsable y comprometido, lo que requiere un enfoque holístico de modo que avance en la concepción de su trabajo para un territorio sostenible. 
Así, las metodologías activas aplicadas en la enseñanza-aprendizaje de la asignatura parten, de la filosofía constructivista, donde "los estudiantes son el eje y los protagonistas del proceso, y son quienes deciden cuándo y cómo aprender, mientras que el profesor es sólo un guía que orienta, motiva y retroalimenta a los estudiantes" (Caro y Reyes, 2003: 49). Enfoque este que alumbra y es aplicado en todas las fases de trabajo de la asignatura.

Siguiendo en esta línea, y dado el objeto de análisis en la asignatura (el municipio) dentro del marco de la educación para la sostenibilidad, así como los interlocutores con los que interactúa finalmente el estudiante, se trabaja desde otros tres métodos de enseñanza aprendizaje:

El aprendizaje servicio, en tanto en cuanto uno de los objetivos de la materia es abrir la Univerisidad a la sociedad, que nuestros estudiantes tengan un contacto directo con la realidad social y laboral dentro de su campo disciplinar, el trabajo ético y auto responsable y autoconsecuente, así como que los resultados de su trabajo tenga utilidad para la Administración. Recogiendo aquí las ideas de Aramburuzabala, Cerrillo y Tello (2015: 85), este tipo de aprendizaje tiene un "carácter experiencial que integra el servicio a la comunidad y la reflexión crítica con el aprendizaje académico, el crecimiento personal y la responsabilidad cívica".

A ello se une la modalidad de aprendizaje por ensayo y error, donde el estudiante sustituye el aprendizaje de fórmulas por el planteamiento de problemas, de modo que puede extraer interrelaciones y sinergias entre variables de análisis e intervención, cultivando de esta forma el pensamiento divergente y la creatividad (Sosa, A. 2001).

Así mismo, para que el alumnado integre un enfoque holístico, siga trabajando el pensamiento divergente y la creatividad en las propuestas, y que sus resultados tengan reflejo sobre la sostenibilidad muncipal, es necesario el aprendizaje colaborativo y la interacción grupal, utilizando para ello herramientas dialógicas: diálogo, debate y discusión.

\section{Fundamentos docentes $y$ fases}

Las aplicación de las metodologías de enseñanza-aprendizaje comentadas en las líneas anteriores, se realiza a través de un proceso que se ha estructurado en cuatro fases. Estadios estos que nos permiten describir y comprender la dinámica que se produce en el aula y que finaliza en la elaboración de una Auditoria Ambiental municipal:

1. Fase de contrucción del conocimiento

2. Fase de desarrollo de indicadores

3. Fase de dinámica grupal participativa

4. Fase de revisión y transferencia a la sociedad 
Se llama la atención aquí, antes de continuar, que el protagonismo de las diferentes metodologías señaladas, a lo largo de estas fases, va variando en adaptación al número de estudiantes participantes en cada curso, que han variado notablemente, desde 33 el primer año que se impartió la asignatura (hace cinco años) hasta 6 estudiantes en la última edición de la misma. Todo ello derivado de una reducción sustancial en la matrícula de los estudios de Ingenería de Obras Públicas.

En la siguiente tabal se indican los municipios sobre los que se ha aplicado esta metodología, población, curso y número de estudiantes.

Tabla 1.Municipios sobre los que se ha desarrollado la Auditoría Ambiental.

Fuente: elaboración propia

\begin{tabular}{|l|c|c|c|}
\hline MUNICIPIO & POBLACIÓN (hab) & CURSO & N $^{\mathbf{0}}$ ESTUDIANTES \\
\hline Vila-real & 50.450 & $2013-14$ & 33 \\
\hline La Vall d'Uixó & 32.655 & $2015-16$ & 19 \\
\hline Tavernes de la Valldigna & 18.015 & $216-17$ & 6 \\
\hline Almassora & 25.648 & $2017-18$ & 6 \\
\hline
\end{tabular}

A continuación, se describirán con más detalle cada una de estas fases.

\subsection{Fase de contrucción del conocimiento}

Es la fase de toma de contacto con los estudiantes en la cual se introducen conceptos nuevos, relacionados con la sostenibilidad, que serán de aplicación en el trabajo de Auditoría Ambiental. Este proceso no tiene como protagonista al profesor sino que se hace participe a los estudiantes desde el primer momento, ya que los conceptos que serán necesarios para el desarrollo de la actividad los construyen los estudiantes, siendo el profesor el guía en el proceso. La forma de proceder consiste básicamente en preguntar al estudiante qué entiende por un determinado concepto, a partir de esta cuestión se genera una reflexión por grupos reducidos, y se exponen sus diversas opiniones mediante definiciones y palabras clave. Todas las definiciones se agrupan en una diapositiva dando lugar a un debate entre los estudiantes con la participación puntual del profesor. Posteriormente se expone por el profesor las ideas fuerza que han sido desarrolladas sobre el conocimiento de la materia por el mundo científico.

Con este proceso el estudiante se introduce activamente en la creación y adquisición del conocimiento.

En esta fase, adicionalmente, se llevan a cabo exposiciones individuales sobre lecturas de documentos y análisis de los objetivos de desarrollo sostenible, de modo que son los estudiantes los que introducen a sus compañeros nuevos conocimientos que nececesitan aprender y reflexionar previamente al desarrollo del trabajo de Auditoría Ambiental. A 
partir de ello, se generan debates y discusiones que permiten construir el conocimiento de la materia objeto de la asignatura.

\subsection{Fase de desarrollo de indicadores}

En esta fase se explica y define el alcance del trabajo, debiendo elaborarse por parte del estudiante, una batería de indicadores, que según el número de participantes, se realiza de manera individual o por grupos de entre 3 a 6 estudiantes (Fig.1). Los indicadores objeto de esta fase se construyen conforme a la metodología propuesta por el Ministerio de Medio Ambiente, Medio Rural y Marino, a través de la guía denominada "Sistemas de indicadores y condicionantes para ciudades grandes y medianas". Así mismo, se han utilizado diversas fuentes bibliográficas que ayudan al estudiante a comprender el significado y alcance de los indicadores construidos, como la publicación de Salvador Rueda "El urbanismo ecológico".

Si bien en esta fase destaca el trabajo individual, resulta necesario interactuar con el resto del equipo (estudiantes) debido a que parte de la información que se utiliza es común y compartida lo que requiere comunicación y coordinación entre los mismos.

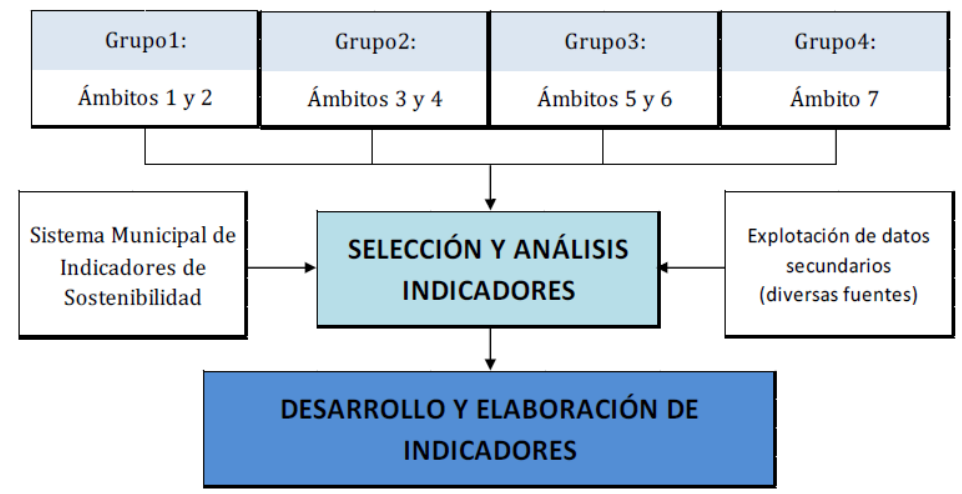

Fig. 1. Grupos de trabajo en la elaboración de indicadores en la Auditoría Ambiental de Vila-real. Fuente:

Elaboración propia.

Cabe destacar también que en esta fase es cuando se produce una interacción mayor con los técnicos municipales del Ayuntamiento objeto de estudio, debido a la necesidad de adquirir datos e información diversa que puede contribuir a la elaboración de los indicadores y su análisis.

Es importante destacar que durante este proceso se programa una visita de campo guiada por profesores y técnicos municipales que ayudan al estudiante a potenciar su conocimiento 
de la ciudad y comprender mejor los resultados que se obtengan en el cálculo de indicadores, mejorando de manera sustancial el diagnóstico que hacen de los mismos.

\subsection{Fase de dinámica grupal participativa}

En esta fase se integran los indicadores desarrollados por los estudiantes, con el objeto de elaborar un diagnóstico municipal sobre aspectos ambientales, sociales y económicos mediante dinámicas grupales basadas en el debate, la crítica y la reflexión colectiva. Con estas técnicas se busca poder concretar los aspectos positivos y negativos, tanto internos como externos, que caracterizan al municipio objeto de estudio, que permitan concluir el diagnóstico municipal de forma consensuada y abierta por todos los participantes (estudiantes), actuando el profesor como dinamizador de la actividad (Fig. 2).

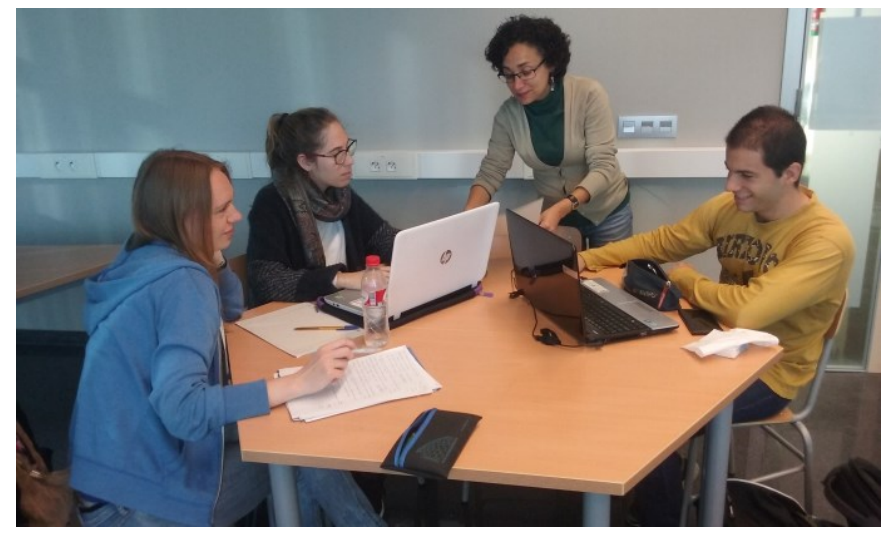

Fig. 2. Mesa de trabajo durante la dinámica grupal en la fase de diagnóstico. Fuente: Elaboración propia.

Este estadio del trabajo de los estudiantes es fundamental, ya que no sólo supone alcanzar un diagnóstico municipal consensuado entre todos, sino que a su vez se construyen las sinergias, es decir, los efectos que el estado de un indicador y una varible genera sobre otros indicadores y variables (Fig. 3). Importantísimo a la hora de que el alumnado interiorice la característica sistémica del territorio, y la necesidad de un análisis y visión integral en la construcción del camino hacia la sostenibilidad municipal. 
Taller 1

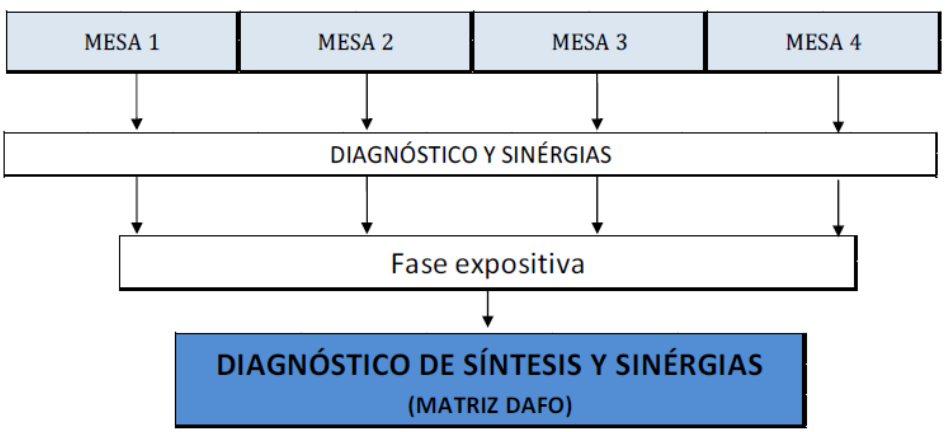

Fig. 3. Organización de los Talleres en la fase de diagnóstico y sinergias. Fuente: Elaboración propia.

Una vez consensuado el diagnóstico municipal, comienza la etapa propositiva donde, siguiendo el marco de aplicación de las metodologías activas descritas, y fundamentalmente, dentro de la colaboración y trabajo grupal o de equipo, los estudiantes generan sugerencias de acción, incorporando sinergias y efectos multiplicadores, que son debatidas, reflexionadas y criticadas (dialógicas) por ellos mismos, siendo el papel del profesorado el de guía, orientador y moderador. Estas propuestas suponen la asunción de un compromiso y responsabilidad, que debe asumir el estudiante, a fin de corregir los aspectos negativos derivados del diagnóstico anterior, y potenciar los positivos, es decir, orientar al Consistorio sobre cuestiones que deberían ser consideradas para potenciar el camino hacia la sostenibilidad municipal. Para finalizar este proceso se propone una priorización, consensuada entre los participantes, de las sugerencias elaboradas, cuyo resultado es su ordenación jerárquica (Fig. 4).

$\underline{\text { Taller } 2}$

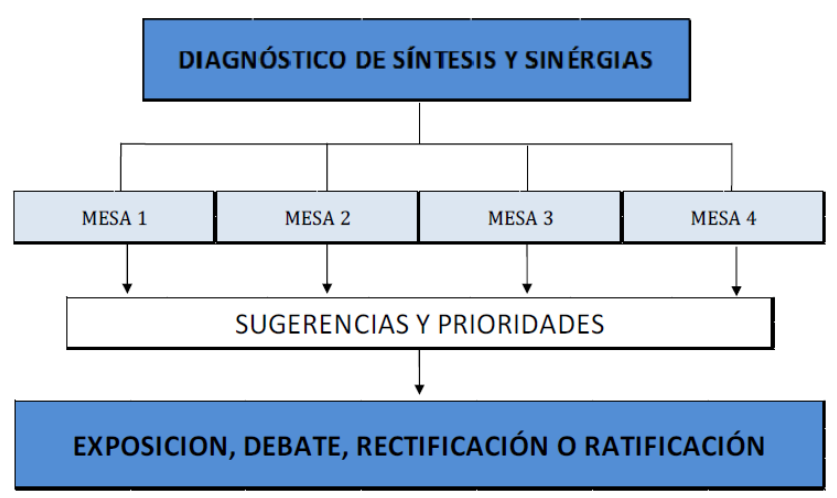

Fig. 4. Organización de los Talleres en la fase de sugerencias y prioridades. Fuente: Elaboración propia. 


\subsection{Fase de revisión y transferencia a la sociedad}

Una vez finalizada la elaboración de la Auditoría Ambiental por parte de los estudiantes, esta es revisada por los profesores que han orientado y dinamizando los talleres que se han realizado para la elaboración de la misma. En esta fase se llevan a cabo aquellas correcciones que procedan y se prepara al alumnado participante para su presentación ante el Ayuntamiento, es decir para devolver los resultados obtenidos a la sociedad, en un acto abierto a la ciudadanía, y desarrollado ante autoridades locales (Alcalde/sa y concejales/as) y técnicos municipales fundamentalmente (Fig.5), de modo que, aposteriori de la presentación, se abre un espacio de debate, crítica y reflexión con los interlocutores presentes. Además, generalmente se encuentra cubierto por la prensa local y/o comarcal que informa sobre el acto, lo que supone la repercusión mediática del acto, con el consecuente estado de responsabilidad y compromiso para los estudiantes, que sin duda alguna, en todas las ediciones han superado exitosamente esta fase de presentación.

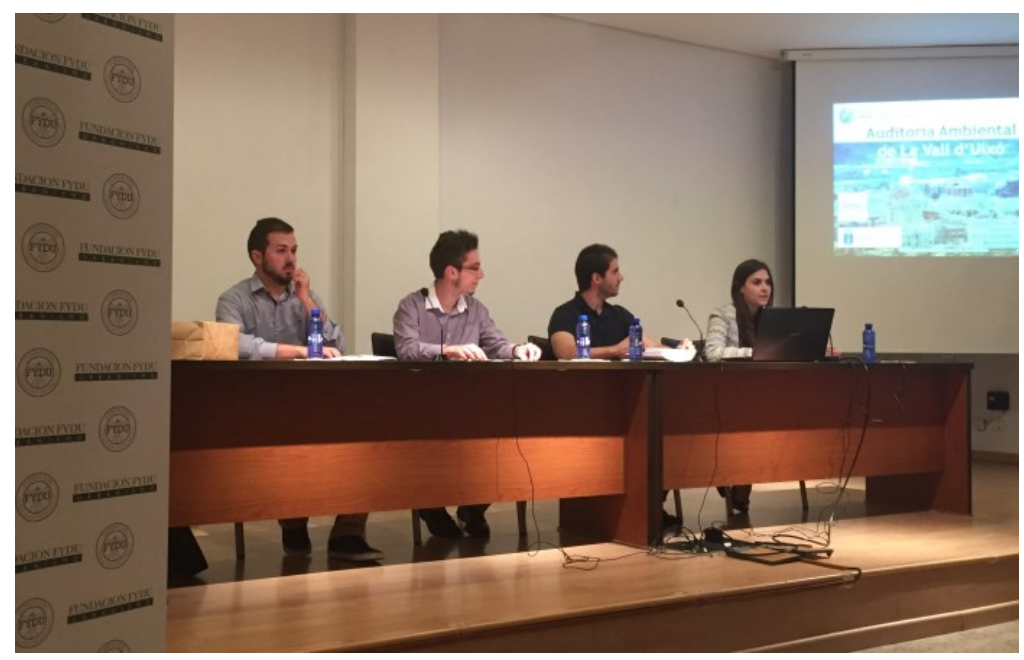

Fig. 5 Presentación de la Auditoría Ambiental en la Vall d’Uixò (Mayo de 2016). Fuente: Elaboración propia.

En las últimas ediciones, el trabajo de Auditoría Ambiental ha sido acompañado de unos paneles, en los cuales se resume el análisis, diagnóstico y sugerencias, contribuyendo a que los asistentes puedan visualizar dichos resultados, e y interactuar de forma mas directa y personal con los estudiantes, comentando aquellas cuestiones que el público mostrase mayor interés (Fig.6). Todo ello como una parte más de la misión de transferencia devolución de resultados- que asume la Universidad. 


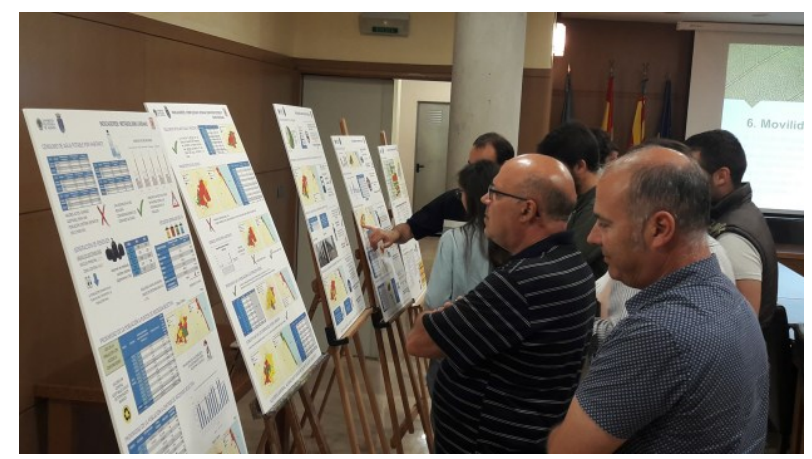

Fig. 6 Exposición de paneles resumen de la Auditoría Ambiental en Tavernes de la Valldigna (Mayo 2017). Fuente: Elaboración propia.

\section{Resultados}

Con las actividades que han sido llevadas a cabo y la metodología propuesta se han observado en las cinco ediciones en las que se ha desarrollado este trabajo (cuatro de ellas presentado en municipios) una serie de resultados que conviene destacar:

1. Los estudiantes han ampliado y mejorado el lenguaje propio de la ingeniería civil y en particular en materia de sostenibilidad de las ciudades.

2. El aprendizaje autónomo de conocimientos con la dinamización de los profesores ha resultado exitosa, observándose que el interés e implicación del estudiante con la materia es mayor que si la formación se basa en lecciones magistrales.

3. El hecho de trabajar un caso real, con la exigencia de trasladar los resultados ante la sociedad civil en un acto público, supone que el alumando se implique con mayor rigor en el desarrollo de la Audotoría Ambiental e interiorice los conocimientos adquiridos y construidos en las primeras fases del trabajo.

4. El estudiante aprende a desarrollar el pensamiento divergente y el análisis crítico sobre los indicadores que ha determinado, cuestionándose su significado y el de los valores obtenidos, así como las acciones que podrían ser llevadas a cabo para revertir una situación que se dirige hacia la insostenibilidad.

5. A raíz de la reflexión sobre sinergias o efectos multiplicadores a partir de la situación actual y las sugerencias de actuación que construyen, el alumnado termina la interiorización del carácter sistémico socio-territorial y la necesidad del trabajo grupal o de equipo para alcanzarlo.

En cualquier caso, quizás el resultado más significativo tiene que ver con la interacción con las administraciones locales: la exposición y presentación de la Auditoría Ambiental ante los representantes mucicipales y técnicos constituye un reto importante para el estudiante y 
los miembros de la corporación local y asistentes al acto, los cuales reciben muy positivamente el trabajo desarrollado, así como el diagnóstico realizado. En este ejercicio, el estudiante demuestra haber desarrollado una comprensión exhaustiva de los problemas ambientales, sociales y económicos que presenta la ciudad y sobre todo, consiguiendo transmitirlos de forma coherente y con el lenguaje apropiado de la ingeniería a la sociedad civil. En todas las presentaciones realizadas la prensa local ha emitido un comunicado de prensa sobre los resultados presentados de la Auditoría Ambiental, como el ejemplo que se observa en la figura 7, en el municipio de Almassora.
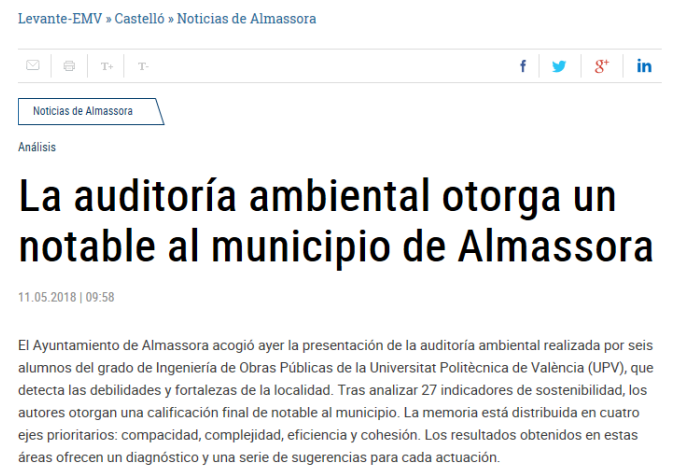

Fig. 7 Nota de prensa sobre la presentación de la Auditoría Ambiental de Almassora (Mayo 2018). Fuente:

Diario Levante-EMV.

\section{Conclusiones}

La experiencia que los estudiantes tienen con una actividad docente, organizada desde principios constructivos, por tanto con herramientas de aprendizaje activo, como la descrita en los apartados anteriores fomenta que los mismos adopten una actitud de mayor implicación con el trabajo que desarrollan, si desde el primer día son conocedores de los objetivos que se pretenden.

Asimismo, aprenden a desarrollar un trabajo que se aproxima a un trabajo profesional, descubriendo en la mayoría de los casos que la información necesaria no se encuentra siempre disponible en las fuentes de información, debiendo ser conocedores de las limitaciones que los informes que se elaboran presentan en muchas ocasiones.

La limitación del tiempo en esta materia condiciona el no poder profundizar en el aprendizaje, especialmente en las dinámicas grupales, debido a que la mayor parte del tiempo se dedica a la búsqueda de información, al cálculo de indicadores y elaboración de fichas explicativas de los mismos, llegando en ocasiones con el tiempo muy ajustado a las actividades de discusión y diagnóstico. 


\section{Referencias}

Aramburuzabala, P.; Cerrillo, R.; Tello I. (2015). “Aprendizaje-servicio: una propuesta metodológica para la introducción de la sostenibilidad curricular en la Universidad”. En Profesorado. Revista de currículum y formación de profesorado, Vol. 19, nº 1 p. 78-95.

Caro Spinel, S. y Reyes Ortiz, J.C. (2003). Prácticas docentes que promueven el aprendizaje activo en ingeniería civil". En Revista de Ingeniería, ${ }^{\circ}{ }^{18}$, p. 48-55.

Ministerio de Fomento y Agencia de Ecología Urbana de Barcelona (2014). Certificación del urbanismo ecológico. Madrid: Ministerio de Fomento y Agencia de Ecología Urbana de Barcelona. Gobierno de España.

Ministerio de Medio Ambiente, Medio Rural y Marino (2011). Sistemas de indicadores y condicionantes para ciudades grandes y medianas. Madrid: Ministerio de Medio Ambiente, Medio Rural y Marino. Gobierno de España.

Rueda, S. (2013). El urbanismo ecológico. Barcelona: Diputación de Barcelona.

Sosa Espinosa, A. (2001) "Sociología como optativa de primer ciclo, en la E.T.S.I. Caminos, Canales y Puertos. Una experiencia docente". I Jornadas de Innovación educativa en la Universidad Politécnica de Valencia. Libro de Resúmenes. UPV-VICAA

Levante El Mercantil Valenciano (2018, 11 de mayo). Noticia publicada en el siguiente link https://www.levante-emv.com/castello/2018/05/11/auditoria-ambiental-otorga-notable$\underline{\text { municipio/1716386.html }}$ 